\title{
Altered human cytomegalovirus-encoded miRNAs in host circulation: novel disease biomarkers and potential aetiological agents
}

\author{
Cheng Wang, Jun-Jun Wang ${ }^{*}$ and Chunni Zhang ${ }^{*}$
}

\begin{abstract}
Circulating microRNAs (miRNAs) are exceptionally stable molecule markers in extracellular environments for various diseases. Importantly, some circulating miRNAs that were encapsulated in extracellular microvesicles (MVs) have been identified as critical mediators of intercellular communication, and function as novel cell-cell crosstalk generegulators. Burgeoning evidence has demonstrated that several types of viruses, including the human cytomegalovirus (HCMV), can encode various miRNAs that play essential roles in disturbing the translation of either the eukaryotic host's genes or virus own during multiple pathophysiological processes. Recently, HCMV-encoded miRNAs have also been uncovered in human circulation, moreover, some circulating HCMV-encoded miRNAs showed specific expression patterns in different diseases with no precise aetiology. In particular, dysregulated HCMV-encoded miRNAs can effectively regulate the host genes regulation, and were implicated in disease development. Given the clinical impact of circulating miRNAs and their abilities to profoundly modulate specific recipient cells, we postulate that characterization of altered HCMV-encoded miRNAs in host circulation may afford valuable insights into developing non-invasive diagnostic biomarkers and clarifying the potential pathophysiological mechanism for various complex diseases, even if the research on circulating HCMV miRNAs is just emerging. The focus of this review is on summarizing the updates on current developments and perspectives for diagnostic and discriminative usefulness of circulating HCMV-encoded miRNAs in various diseases, including essential hypertension, oral lichen planus disease, chronic hepatitis B and type 2 diabetes. We also review the physiological and pathophysiological effects of HCMV-encoded miRNAs on disease development and progression.
\end{abstract}

Keywords: microRNAs, Cytomegalovirus, Biomarker, Extracellular vesicles, Exosome

\section{Introduction}

MicroRNAs (miRNAs) are a family of $19 \sim 24$ nucleotide, single strand small non-protein coding RNAs that play pivot roles in inhibiting human gene expression at the post-transcriptionally level. Till date, over 2000 of miRNAs in human beings have been predicted and verified, which were estimated to disturb the translation of up to $60 \%$ of human protein-encoding genes $[1,2]$. Since lin-4, the first miRNA was identified in C. elegans in 1992, extensive studies from the last two decades have

\footnotetext{
*Correspondence: wangjunjun9202@163.com; zchunni27@hotmail.com Department of Clinical Laboratory, Jinling Hospital, State Key Laboratory of Analytical Chemistry for Life Science, Nanjing University School of Medicine, Nanjing University, 305 East Zhongshan Road, Nanjing 210002, China
}

documented that miRNAs have potent functions in regulation of virtually all biological processes, including organisms development, differentiation, and cellular function, while dysregulated miRNAs were uncovered to be implicated in several pathological processes and development of disease, including virus infectious diseases $[3,4]$. More importantly, several distinct miRNA expression signatures have been reported in different infectious diseases $[5,6]$. Thus, miRNAs are recognized as a promising novel class of molecular biomarkers for human infectious diseases, especially for virus infection.

In 2008, our groups and others firstly found that miRNAs are also steadily existed in the circulation and may be utilized as minimal-invasive unique molecular

(c) The Author(s). 2019 Open Access This article is distributed under the terms of the Creative Commons Attribution 4.0 International License (http://creativecommons.org/licenses/by/4.0/), which permits unrestricted use, distribution, and reproduction in any medium, provided you give appropriate credit to the original author(s) and the source, provide a link to the Creative Commons license, and indicate if changes were made. The Creative Commons Public Domain Dedication waiver (http://creativecommons.org/publicdomain/zero/1.0/) applies to the data made available in this article, unless otherwise stated. 
markers for the diagnosis of human disease [7]. Till date, a large body of research has identified aberrant circulating miRNA expression profiles in various diseases and showed those signatures were tightly correlated to disease classification, diagnosis, progression and prognosis. Intriguingly, by utilizing a combination of computational methods, next-generation sequencing technology, northern blot hybridizations and the qRT-PCR technologies, an enriched virus-encoded miRNAs were also discovered in the circulation of subjects with virus infection [8-10]. More importantly, the presence of circulating virus miRNAs seems to have significant clinical value $[8,11]$. For instance, Epstein-Barr Virus (EBV) can encode their own miRNAs, and up to 44 mature EBV-encoded miRNAs have been reported currently [12-14]. Remarkably, several EBV miRNAs can be directly detected in blood circulation and showed dysregulation in human hematologic malignancies $[8,11,15]$. In addition, altered circulating EBV miRNAs were also reported in several solid tumors, such as nasopharyngeal cancer and gastric carcinoma, and may serve as useful biomarkers of EBV-related malignancies and chronic active EBV infection [16-18]. Apart from circulating EBV-encoded miRNAs, other virus including Kaposi sarcoma-associated herpesvirus (KSHV), BK virus (BKV), Polyomavirus JC (JCPyV), and Papillomaviruses (PVs) derived miRNAs in blood circulation were also found to be a hopeful approach for the exploitation of disease markers $[6,10,19-22]$. Recently, Chen and colleagues reported that several miRNA-like RNA fragments can be predicted in Ebola virus genome and they successfully confirmed the existence of miR-VP-3p in the serum samples of patients with Ebola virus disease (EVD), moreover, monitoring the levels of serum miR-VP-3p may use as an early indicator of EVD [23]. Collectively, these observations point out a novel vision for the use of viral encoded miRNAs as minimal-invasive markers for diagnosing, outcome evaluation, and therapeutic effectiveness assessment of virus associated diseases.

Despite recent advances, studies on the circulating virus-encoded miRNA and their clinical relevance remain sparse. Like EBV and KSHV, the human cytomegalovirus (HCMV) can also maintain a persistent or latent infection during the lifespan of the natural host [24]. HCMV is a widespread beta-herpesvirus that can induce severe illnesses in immunologically immature or compromised individuals. Similar to the EBV and KSHV can encode their own miRNAs, to date, HCMV has been reported to encode 24 miRNAs [25]. Notably, HCMV-encoded miRNAs have also been reported to participate in viral infection by playing life cycle-specific roles [26]. Even though the validated downstream target genes and the pathophysiological functions of most HCMV-encoded miRNAs remain elusive, intensive functional investigations have demonstrated that they were participated in modulating HCMV latent infection and disturbing the host immune responses [2730]. Moreover, HCMV-encoded miRNAs were also found to directly influence the expression of host genes and involved in the pathological processes of health-threatening diseases [31, 32]. Very recently, our lab and others have demonstrated the presence of HCMV miRNAs in blood circulation, more importantly, the expression pattern of serum HCMV miRNAs exhibit obvious difference in patients with essential hypertension, oral lichen planus disease, chronic hepatitis B, acute aortic dissection, and diabetes when compared with healthy controls. Furthermore, mechanism studies indicate a causal link between altered HCMV miRNAs in circulation and their pathogenic roles in the diseases promotion. These discoveries raised the intriguing possibility that the levels of specific circulating HCMV-encoded miRNAs may serve as novel informative diagnostic tool for diseases and virological outcomes, and also provide indicative information about HCMV infection involvement in diseases pathogenesis. Remarkably, accumulated findings have convincingly proved that miRNAs in circulation can be selectively encapsulated in exosomes and shedding vesicles, and these vesicles can behave as extracellular carriers of circulating miRNAs that can transferred to neighboring cells and modulating the recipient's gene expression [33]. More interestingly, recent studies revealed that viruses can hijack the exosome pathway, and virus-encoded miRNAs can be actively secreted via exosomes from virus-infected cells and delivered to and act in virus-negative recipient cells [34]. Furthermore, our latest study implied that specifically altered plasma HCMV miRNA is primarily encapsulated in exosomes [35]. Thus, taken together our findings combined with those previous reports, the exogenous HCMV-encoded miRNAs can be packaged into exosomes and delivered to recipient cells, moreover, the involvement of exosome-encapsulated HCMV-encoded miRNAs in host recipient cellular gene repression may also provide further insights into novel and somewhat surprising potential therapeutic targets.

The present review will summarize the presence, biological roles, and clinical significance of HCMV-encoded miRNAs with emphasis on the current progresses of the altered HCMV-encoded miRNAs in host circulation. An exhaustive understanding of the modulatory roles of HCMV-encoded miRNAs will shed light on exploring the complicated HCMV-host interactions and raise novel diagnostic and treatment strategies involving HCMV-encoded miRNAs.

\section{HCMV-encoded miRNA biogenesis}

Existing evidence demonstrated that the HCMVencoded miRNA biogenesis has no obvious difference from that of cellular miRNAs [36-38]. Briefly, the biosynthesis of HCMV-encoded miRNA initials as 
single-stranded pri-miRNAs transcribed in the nucleus by host RNA Pol II which are recognized and processed by the host nuclear RNaseIII endonuclease enzyme Drosha to produce a stem-loop pre-miRNA with the length around 70 nucleotides. Pre-miRNAs are subsequently exported to the cellular cytoplasm and then cleaved into short, 22 nucleotides duplex RNAs by the host RNaseIII endonuclease enzyme Dicer. Of the two strands of the duplex, one strand may be incorporated into an Argonaute protein containing effector complex known as RISC, and the other strand from the corresponding arm of the stem loop, known as the "miRNA-star", is recognized to be rapidly degraded. The incorporated miRNA functions as a guide sequence directs RISC to identify target mRNAs and leads to post-transcriptional regulation of target gene.

\section{Expression profile of HCMV-encoded miRNAs in HCMV- infected cell lines and patients}

By employing a combination of direct small RNAs cloning, algorithm prediction, deep sequencing, and northern blotting, a series of studies have characterized the HCMV miRNAs expression profiles in HCMV-infected cell lines and patients. In 2005, Pfeffer and colleagues firstly demonstrated that HCMV was capable of generating miRNAs, and they successfully identified nine HCMV miRNAs from lytically infected primary human fibroblast [39]. Subsequently, Grey et al. predicted and characterized the HCMV miRNAs in HCMV AD169 infected primary fibroblast cells [40]. By examining the RNA species of HCMV-infected fibroblast cells, they found that 5 HCMV-encoded miRNAs, including miR-UL36-1, miR-US5-1, miR-US5-2, miR-UL70-1 and miR-US4-1, were highly expressed during infection. Notably, three of the five HCMV miRNAs were also identified in Pfeffer et al. study [39, 40]. Meanwhile, Walter Dunn and colleagues characterized HCMV miRNA expression profiles of HCMV infected human foreskin fibroblasts (HFFs) and astrocytoma cells by using small RNA deep sequencing, in silico analysis and northern analyses [41]. Consequently, they successfully identified three novel HCMV-encoded miRNAs, including miR-UL23-5p, miR-UL23-3p and miR-US24, which were exclusively abundant in HCMV-infected cells. Additionally, the three miRNAs also showed consensus expression levels in both a laboratory strain and a clinical low-passaged isolate, which indicates that these three miRNAs may directly implicate in HCMV-associated infection and pathogenicity [41]. To increase the understanding of the HCMV-encoded miRNAs, Stark et al. performed a next generation sequencing to investigate the small RNAs signatures of HCMV-infected fibroblast cells. Likewise, they successfully identified $22 \mathrm{HCMV}$-encoded miRNAs, including two novel miRNAs, miR-US22 and
miR-US33as, in the HCMV-infected fibroblast cells [25]. Additionally, these miRNAs were capable of inhibiting host targets in vitro. Besides fibroblasts, aberrant HCMV miRNA expression pattern was also observed in HCMV-infected THP-1 cells [42, 43]. Fu et al. analyzed the small RNAs in HCMV-infected THP-1 cell line, and observed that miR-US25-1, miR-US25-2-5p and miR-UL112 were expressed during HCMV latent infection [42]. Notably, RNA sequencing analysis revealed that miR-US25-1 and miR-US25-2 were the most enriched miRNA of the total HCMV miRNAs reads, which indicated that these two miRNAs may involve in viral latency [42]. Shortly afterwards, Shen et al. examined the HCMV miRNA expression during quiescent, semi-permissive and permissive infection in undifferentiated, differentiated THP-1 monocytes and human embryo lung fibroblasts (HELs), respectively. They found that the expression signatures for $16 \mathrm{HCMV}$-encoded miRNAs showed markedly difference among the three types infected cell. Of the examined miRNAs, fifteen were obviously enriched in HELs during virus lytic infection, fourteen miRNAs showed moderate induction in differentiated THP-1 monocytes, and three miRNAs showed low expression levels in quiescently infected THP-1 cells [43]. Interestingly, only miR-UL70-3p showed poorly induction in HELs, moderately in undifferentiated THP-1 cells, but strongly in differentiated THP-1 cells, which suggesting a special function of miR-UL70-3p in THP-1 and d-THP-1 cells [43].

HCMV-encoded miRNAs were also markedly dysregulated in HCMV-positive patients. The first evidence of HCMV-encoded miRNAs that exhibit statistically alteration was reported in patients with glioblastoma (GBM). Ulasov et al. analyzed the expression profile of HCMV-encoded miRNAs in GBM tissues, notably, the expression levels of CMV70-3p miRNA (miR-UL-70-3p) showed more than 10-fold upregulation in the GBM tissue when compared to control brain tissues. Additional functional studies revealed that overexpression of CMV70$3 p$ can regulate cancer stemness of CD133 positive glioblastoma cell lines, while downregulation of CMV70-3p can abrogate cell migration and invasion of glioma stem cells, which indicating that CMV70-3p may be a contributing factor for GBM [44]. Subsequently, Liang and colleagues found that miR-UL112-3p was also markedly increased in GBM, and elevated miR-UL112-3p levels were significantly correlated with tumor volume, differentiation grade, clinical stage, and prognosis of GBM patients. In particular, miR-UL112-3p could regulate the biological behaviors of GBM cells including cell proliferation, clone formation, migration, invasion, and represented as an "oncomir" by promoting GBM tumorigenesis [45]. The upregulation of miR-UL112-3p in GBM was further confirmed by a latest study. Deshpande et al. demonstrated 
that the expression of miR-UL-112-3p were markedly increased in GBM tissues and may participate in virus immune escape, as well as the GBM immune microenvironment modulation [46]. Dysregulated HCMV miRNAs were also observed in the human dental pulp and gingiva tissues. Zhong et al. examined the expression profile of virus-encoded miRNAs in normal and diseased pulps, and identified that $2 \mathrm{HCMV}$ miRNAs including miR-US4 and miR-UL70-3p were obviously elevated 4.64-, and 5.36-fold, respectively [47]. Bioinformatics analyzes suggest that the differentially expressed viral miRNAs may involve in targeting inflammation, immunity, and angiogenesis related genes of host [47]. In the meanwhile, follow-up study confirmed that the expression levels of miR-US4 were also increased in gingival biopsies that collected from periodontitis patients [48]. Together, these results demonstrated that HCMV miRNAs were widely expressed in several types of tissues and some were significantly altered in tissues from patients, and thus play key roles in disease development.

Till date, a total of $26 \mathrm{HCMV}$-encoded miRNAs were deposited in the miRbase 22.0, and most of them have been verified in HCMV infected cells and tissues, nevertheless, the list continues to expand. In addition, until now, the HCMV miRNAs expression patterns have been comprehensively examined in limited HCMV transformed cell lines and only a few of altered miRNAs were reported to contribute toward pathogenesis in patients. Therefore, the defining characteristics of HCMV miRNAs in other HCMV-susceptible cell lines as well as HCMV-associated disease remains to be elucidated.

\section{Biological and pathophysiological functions of HCMV miRNAs}

Currently, the biological and pathophysiological functions of most HCMV miRNAs are largely unclarified, however, empirical evidence demonstrates that some HCMV-encoded miRNAs play crucial roles in modulating the viral life cycle as well as the crosstalk between viruses and their hosts. One important role of HCMV-encoded miRNAs is to facilitate HCMV-infected cells to escape recognition from the host immune system, thus contributing to immunoevasion. Stern-Ginossar et al. applied a bioinformatics method to analyze the downstream genes that regulated by HCMV miRNAs, and successfully identified the major histocompatibility complex class I-related chain B (MICB) as a potential target gene of miR-UL112. Functional study demonstrated that miR-UL112 can directly downregulate MICB translation during viral infection, thus resulting in the reduced elimination of HCMV-infected cells by NK cells [49]. Inhibition of MICB translation by miR-UL112 was confirmed by another similar study [27]. Most notably, miR-UL112 can function synergistically with the host miRNA to repress MICB translation, and this newly discovered viral miRNA-host miRNA interaction may facilitate the HCMV to escape elimination by host's immune cells during virus infection [27]. Moreover, miR-UL112 may promote HCMV immune evasion through post-transcriptional regulation of Interleukin-32 (IL-32) [50]. IL-32 is an important cytokine in innate and adaptive immune responses, which is induced by viral infection and plays crucial roles in host defense against viral infections. The expression of IL-32 could be functionally attenuated by overexpressed miR-UL112, and down-regulation of IL-32 might modulate NK cell activation through the TNF- $\alpha$ pathway, thus achieving HCMV immune evasion [50]. Additionally, other studies also demonstrated that HCMV miRNAs implicated in virus immune evasion by targeting ERAP1, an important factor that trims precursors into peptides of the correct length to bind MHC class I molecules. For instance, Kim et al. showed that miR-US4-1 directly inhibited ERAP1 expression during viral infection, which resulted in the infected cells avoiding immune clearance from HCMV-specific cytotoxic T lymphocytes [29]. Recently, Romania et al. confirm that miR-UL112-5p can also target ERAP1, thereby disturbing the presentation process of the HCMV pp65 $495-503$ peptide to specific CTLs, and leading HCMV immune evasion [51].

HCMV miRNAs may act as crucial regulators for viral replication. As described above, miR-UL112-1 has been found to participate in promoting immune evasion through targeting MICB as well as MICB modification associated ERAP1. Moreover, miR-UL112-1 was also involved in viral replication. By utilizing a bioinformatics analysis and luciferase assays, three viral transcripts, such as trans-activating factor IE72, were identified as potential targets of miR-UL112-1 [24]. Existing evidence revealed that inhibition of IE72 in the context of low multiplicity infections leads to an obvious attenuation of viral replication, which revealing that overexpression of miR-UL112-1 would inhibit HCMV replication. Remarkably, the miR-UL112-1-mediated inhibition of viral replication and viral titers can also achieve by regulating the UL114 gene, of which is associated with HCMV DNA synthesis [52]. Besides miR-UL112-1, overexpression of miR-US25-2 and miR-US25-1, can also significantly reduce viral DNA synthesis by probably target the host genes that are related with virus DNA replication, even though the exact target genes are not clarified [52]. Interestingly, following mechanism study showed that the eukaryotic translation initiation factor 4A1 (eIF4A1) is a specific target gene of miR-US25-2-3p. Upregulation of this miRNA will knockdown of the protein eIF4A1 expression, and decrease the HCMV DNA synthesis in MRC-5 cells in vitro. However, elevation of eIF4A1 by transient transfection of miRNA inhibitor in MRC- 5 cells results opposite effects [53]. On the other hand, systematic miRNA target screening strategy using RISC immunoprecipitation and RNA interference demonstrated that ATP6VOC is an important component for HCMV replication and can be 
inhibited by miR-US25-1 [54]. Moreover, another study also confirmed that miR-US25-1-5p can reduce HCMV replication by downregulation of the targets YWHAE, UBB, NPM1, and HSP90AA1 directly or indirectly [55]. In addition, two other HCMV miRNAs, miR-US33-5p and miR-US5-1, were also identified as inhibitors of viral DNA synthesis and viral replication by directly targeting STX3 and Geminin, respectively $[56,57]$.

Alteration of HCMV-encoded miRNAs has important roles for latency control of HCMV. For instance, miR-UL112-1 can inhibit the translation of the latency-associated IE genes. IE1 is a critical controller for viral DNA replication, and which can active the expression of the late genes that encode the viral structural proteins [58]. Given the crucial role of IE1 in initiating the lytic program, it is reasonable that miR-UL112-1 inhibits the expression of IE1 may help for maintaining HCMV latency [59]. The critical role of miR-UL112-1 in regulating HCMV latency was confirmed in a THP-1 cell viral latency model system [60]. By applying a THP-1 cell line model of latency, Lau and collages observed that miR-UL112-1 plays an important function during latency by controlling the expression of viral IE72, since enhanced expression of IE72 product would result in latently infected cells becoming the direct targets for IE72-specific CD8 ${ }^{+}$cytotoxic T-cells [60]. Furthermore, one of our lab's latest work demonstrated that miR-UL148D robustly express at the late stages of latent HCMV infection in host cells and facilitate virus latency by regulating the immediate early response gene 5 (IER5)-cell division cycle 25B (CDC25B) axis in host cells. MiR-UL148D can directly inhibit the translation of IER5 at the post transcriptional level and restored the expression of CDC25B during the establishment of viral latency, since CDC25B plays a significant effect on activating cyclin-dependent kinase 1 [61].

HCMV miRNAs can still control the host secretory pathway during infection. The host secretory pathway acts essential functions in HCMV assembly and release from the host cell [30]. Moreover, specific endocytic proteins such as VAMP3, RAB11A, and SNAP23 in the secretory pathway were involved in IL-6 and TNF-a secretion from the host cells, and which can trigger cellular innate immunity. Existing evidence has reported that multiple HCMV-encoded miRNAs can downregulate the protein levels of VAMP3, RAB5C, RAB11A, SNAP23, and CDC42, which results in inhibiting the trafficking and secretion of pro-inflammatory cytokines and aids the virus to escape immune evasion [30]. On the other hand, inhibition of the endocytic pathway genes may benefit to reconfigurate the secretory pathway to form the virion assembly compartment, thus leading to increased efficiency of infectious particle production [30]. The miR-UL112 has also been recognized to regulate the type I interferons (IFNs) signal pathway. For instance, overexpression of miR-UL112 in PBMC using lentivirus resulted in the inhibition of NK cell cytotoxicity. Additional mechanism study revealed that miR-UL112 downregulates NK cell activity through repressing type I IFNs [62]. Similarly, Toll-Like Receptor (TLR) is another target gene of miR-UL112-3p. TLR2 plays important roles in controlling HCMV infection by activating several signal transduction pathways including the NF- $\mathrm{KB}$ pathway. Landais et al. found that the protein contents of TLR2 were decreased, while the expression of miR-UL112-3p was accumulated at late times during HCMV infection in fibroblasts and THP-1 cells. Transient transfection, reporter gene assay, and western blotting confirmed that TLR2 is a target gene of miR-UL112-3p. Moreover, overexpression of miR-UL112-3p in these cells significantly inhibit the expression of multiple cytokines and the TLR2/NF-kB signaling axis [63]. Another chemokine RANTES also plays pivot roles in attracting immune cells during viral infection and therefore influence the pathogenic consequence of virus infection [64]. Kim and colleagues found that the contents of RANTES were reduced in HFF cells at a later stage after infection with the HCMV clinical strain Toledo, in contrary, miR-UL148D showed markedly elevated expression and inversely correlated the levels of RANTES. Interestingly, miR-UL148D-1 can inhibit the expression of RANTES during HCMV infection, thereby leading to markedly inhibition of RANTES release from CMV infected fibroblasts cells and modulating the host immune system [65]. Additionally, miR-UL148D was found to target ACVR1B and limited pro-inflammatory cytokine secretion in HCMV latent infected primary myeloid cells [66]. Besides miR-UL112-1 and miR-UL148D, a very recent work confirmed that miR-US25-1-5p could attenuate the expression of CD147, which can mediate HCMV-triggered antiviral signaling, and induce HCMV lytic propagation at a low multiplicity of infection [67].

Preventing cell apoptosis is an apparent advantage to viruses that take up persistent or latent infections in long-lived cells. HCMV can also play antiapoptotic role in infected cells. Several HCMV encoded miRNAs can target a variety of pro-apoptotic host genes to prevent cell apoptosis. For instance, the human immediate early gene X-1 (IEX-1) was recognized as a key cellular protein in controlling apoptosis and cellular growth [68]. MiR-UL148D inhibits the translation of IEX-1 at the post-transcription level, and the reduction of IEX-1 exerts anti-apoptotic effects during HCMV infection in vitro [69]. MiR-UL148D downregulates the expression of another pro-apoptotic gene, ERN1. The ERN1 gene was reported to be involved in the initiation of endoplasmic reticulum stress-induced apoptosis, and in silico analyzes predicted that ERN1 was targeted by miR-UL148D [70]. Existing studies have documented that adenine nucleotide translocator 3 (ANT3) can strongly induce apoptosis [71, 72]. ANT3 was confirmed as a direct target gene of miR-UL36-5p. In 
latently infected cells, the expression levels of ANT3 were markedly decreased, and miR-UL36-5p may utilize as an inhibitor of ANT3. Enhanced expression of miR-UL36-5p leads to the downregulation of ANT3 imply that miR-UL36-5p may function as an anti-apoptotic miRNA during actual HCMV infection with the aim to maintain a balance between the host and the virus [73]. Shao et al. more recently reported the directly targeting of Glutaminyl-tRNA Synthetase (QARS) by miR-US4-1. Existing evidence has uncovered the critical role of QARS in cell apoptosis through blocking the apoptosis associated signaling pathway [74]. Unlike the role of other HCMV miRNAs for inhibiting host cell apoptosis, miR-US4-1 was implied in facilitating cell apoptosis and benefiting discharge of infectious virus particles through down-regulation of QARS [74]. Surprisingly, miR-US25-1 can enhance the ox-LDL stimulated apoptosis of human endothelial cells. Previous study revealed that several cellular transcripts containing the miR-US25-1 seed sequences in the $5^{\prime}$-UTR rather than the $3^{\prime}$-UTR, but the underlying implication of this phenomenon was unclear, furthermore, the miR-US25-1 was found to involve in cell cycle control [26]. Fan et al. found that miR-US25-1 levels were elevated in HCMV infected subjects or endothelial cells, consequently, the upregulated miR-US25-1 could attenuate the translation of BRCC 3 by combing with the $5^{\prime}$-UTR of BRCC 3. More importantly, in an in vitro ox-LDL treated endothelial cell model, overexpression of miR-US25-1 using transit transfection with miRNA mimics promoted the ox-LDL-induced apoptosis [75]. Collectively, these results confirmed the crucial role of miR-US25-1 in enhancing the ox-LDL-induced apoptosis of endothelial cells by regulating the expression of BRCC 3. Additionally, Shen and colleague demonstrated that miR-UL112 may enhance the host cells growth and proliferation. In silico analyses indicated that overexpression of miR-UL112 significantly altered several physiological pathways including the mitogen-activated protein kinase and chemokine signaling pathways, etc [31].

Till date, the biological functions and downstream target genes of some HCMV miRNAs were reported (see Table 1), however, the list of HCMV-encoded miRNA continues to expand and an in-depth functional studying is lacking for most, only a limited number of targets have been fully clarified for a few of specific HCMV-encoded miRNAs such as miR-UL112-3p, and miR-UL148D. Thus, the functions as well as physiologically relevant for the vast majority of HCMV-encoded miRNAs are still largely unclear and remain to be elucidated in coming future.

\section{Altered HCMV-derived miRNAs in circulation can be served as novel non-invasive diagnostic biomarkers and potential etiological agent for HCMV associated diseases} The seroprevalence of HCMV in human populations approaches to $90 \%$ in the older adults worldwide, with even higher incidence reported in developing countries
[76]. Primary HCMV infection is usually asymptomatic and can establishes latency in various host cells for life, however, HCMV can result severe and even fatal disease in immunocompromised individuals, newborns, and infants [77]. Current method to discern patients at risk of HCMV complications commonly employ surveillance by HCMV viral load monitoring. Determination of HCMV DNA load in peripheral blood leukocytes (PBLs) are frequently applied to evaluate the treatment effect of antiviral therapies and are also used widely to predict the prognosis, risk and severity of HCMV-associated comorbidities [78]. Nevertheless, a major deficiency of this method is hard to differentiate whether the examined HCMV DNA from the latent virus reservoir or from virions being produced actively by infected cells. Another shortcoming is difficult to extrapolate results from one institution to another [78]. Thus, measurement of HCMV DNA from peripheral blood may have limited usefulness on patient management under complicated clinical settings. In addition to CMV load, detection of antibody against CMV (IgG and IgM) can provide information for past CMV infection (CMV-IgG) and acute or recent infection (CMV-IgM), however, existing studies demonstrated that the positive rates of anti-HCMV IgG/ IgM were virtually universal among patients and healthy subjects, and may not helpful for immunecompromised patients who have an attenuated and delayed antibody production [79]. Other methods for detection of HCMV include virus cultures, direct detection of pp65 antigen in peripheral blood polymorphonuclear leukocytes, and quantification of viral nucleic acid in PBLs by PCR. However, each of these detection methods has its own limitations [80].

The finding that HCMV-encoded miRNAs in blood circulation opens up a new area in HCMV investigation and figures out an ideal choice for diagnostic targets for HCMV infections. Recent publications have provided compelling evidence that HCMV-encoded miRNAs are steadily detected in host circulation, and firmly established the links between alterations of circulating HCMV miRNA and pathological conditions such as essential hypertension, cardiovascular disease, cancer, and diabetes, as well as psychiatric and neurological diseases (see Table 2). This is highlighted by specifically dysregulated viral miRNAs that identified from blood circulation can participate in regulating the host transcriptome. The first evidence for the association between circulating HCMV miRNAs and human disease was discovered in essential hypertension patients [32]. Essential hypertension has been recognized as a critical risk factor for cardiovascular diseases and the main cause of chronic renal failure. Existing studies have documented that essential hypertension may be induced by specific environmental and genetic factors, nevertheless, the precise cause of 
Table 1 Validated targets and proposed functions of HCMV-encoded miRNAs highlighted in this review

\begin{tabular}{|c|c|c|c|}
\hline HCMV-encoded miRNA & Target gene & Functions & $\overline{\text { References }}$ \\
\hline \multirow[t]{2}{*}{ hemv-miR-UL112-3p } & MICB & Immune evasion & {$[27],[49]$} \\
\hline & IL-32 & Immune evasion & {$[50]$} \\
\hline hemv-miR-US4-1 & ERAP1 & Immune evasion & [29] \\
\hline hemv-miR-UL112-5p & ERAP1 & Immune evasion & [51] \\
\hline \multirow[t]{2}{*}{ homv-miR-UL112-3p } & $\mathrm{IE} 72$ & Virus replication & [24] \\
\hline & UL114 & Virus replication & {$[52]$} \\
\hline hemv-miR-US25-2 & Undetermined & Virus replication & {$[52]$} \\
\hline hemv-miR-US25-1 & Undetermined & Virus replication & {$[52]$} \\
\hline homv-miR-US25-2 & elF4A1 & Virus replication & {$[53]$} \\
\hline homv-miR-US25-1 & ATP6VOC & Virus replication & {$[54]$} \\
\hline hemv-miR-US25-1 & YWHAE, UBB, NPM1, HSP90AA1 & Virus replication & {$[55]$} \\
\hline hemv-miR-US33-5p & STX3 & Virus replication & {$[56]$} \\
\hline homv-miR-US5-1 & Geminin & Virus replication & {$[57]$} \\
\hline \multirow[t]{2}{*}{ homv-miR-UL112-3p } & $\mathrm{IE} 1$ & HCMV latency & [59] \\
\hline & $\mathrm{IE} 72$ & HCMV latency & {$[60]$} \\
\hline hcmv-miR-UL148D & IER5 & HCMV latency & [61] \\
\hline \multirow[t]{2}{*}{ hemv-miR-UL112-3p } & Type I IFNs & Host secretory pathway & {$[62]$} \\
\hline & TLR2 & Host secretory pathway & [63] \\
\hline \multirow[t]{2}{*}{ hemv-miR-UL148D } & RANTES & Host secretory pathway & [65] \\
\hline & ACVR1B & Host secretory pathway & {$[66]$} \\
\hline hemv-miR-US25-1 & CD147 & Host secretory pathway & {$[67]$} \\
\hline \multirow[t]{2}{*}{ hemv-miR-UL148D } & IEX-1 & Host cell apoptosis & {$[68]$} \\
\hline & ERN1 & Host cell apoptosis & [70] \\
\hline hcmv-miR-UL36-5p & ANT3 & Host cell apoptosis & [73] \\
\hline hemv-miR-US4-1 & QARS & Host cell apoptosis & [74] \\
\hline hemv-miR-US25-1 & BRCC 3 & Host cell apoptosis & [75] \\
\hline hcmv-miR-UL112-3p & MAPK, Chemokine & Host cell growth and proliferation & {$[31]$} \\
\hline
\end{tabular}

Table 2 Differential expression of circulating HCMV-encoded miRNAs under various disease conditions

\begin{tabular}{|c|c|c|c|c|}
\hline HCMV-encoded miRNA & Sample type & Diseases & Alteration & References \\
\hline \multirow[t]{3}{*}{ hemv-miR-UL112 } & Plasma & Essential hypertension & Upregulation & {$[32]$} \\
\hline & Plasma & Type 2 diabetes & Upregulation & [81] \\
\hline & Plasma & Glioblastoma & Upregulation & [81] \\
\hline $\begin{array}{l}\text { hcmv-miR-US5-1, hcmv-miR-US-5-2-3p, } \\
\text { hemv-miR-UL-22a-5p, hcmv-miR-US25-1-5p, } \\
\text { hcmv-miR-US-25-2-3p, hcmv-miR-US-25-2-5p, } \\
\text { hcmv-miR-US-33-3p, hemv-miR-UL36-5p, } \\
\text { hcmv-miR-UL112-3p }\end{array}$ & Blood & Solid organ transplantation & Upregulation & [83] \\
\hline homv-miR-US25-1-5p, homv-miR-US25-2-5p & Plasma & Congenital CMV infection & Upregulation & [84] \\
\hline hcmv-miR-US33-5p & Plasma & Acute aortic dissection & Upregulation & {$[86]$} \\
\hline hemv-miR-US4-1, hemv-miR-UL-148D & Serum & $\begin{array}{l}\text { IFNa treatment non-responsive } \\
\text { chronic hepatitis B }\end{array}$ & Upregulation & [87] \\
\hline $\begin{array}{l}\text { hemv-miR-UL112-3p, hemv-miR-UL22a-5p, } \\
\text { hemv-miR-UL148d, hcmv-miR-UL36-5p, } \\
\text { hcmv-miR-UL59 }\end{array}$ & Serum & Oral lichen planus & Upregulation & {$[35]$} \\
\hline
\end{tabular}


this disease remains elusive. By characterizing the plasma miRNAs pattern of patients with essential hypertension, $\mathrm{Li}$ and colleagues identified that the plasma concentrations of miR-UL112 were markedly higher in hypertensive patients than that in the control subjects. In addition, they uncovered that IRF-1 was a direct target gene of miR-UL112, and the relationship between IRF-1 dysregulation and hypertension indicating a crucial role of miR-UL112 involved in the pathogenesis of hypertension [32]. Subsequently, the miR-UL112 levels in the plasma/serum were further examined in a cohort of 67 patients with diabetes, glioblastoma and rheumatoid arthritis. In agreement with the results from the first study, elevated contents of circulating miR-UL112 were observed in patients with diabetes and glioblastoma, especially in type 2 diabetes patients, as compared with normal controls. Nevertheless, circulating miR-UL112 concentrations show no markedly difference between rheumatoid arthritis patients and controls [81]. These studies confirmed that the altered circulating miR-UL112 may serve as a novel biomarker for HCMV latency or active replication in various HCMV infected patients.

Cytomegalovirus infection remains the most important infectious factor of markedly morbidity, and occasional mortality in patients after solid organ transplantation (SOT). In the current era, several methods such as serology, culture, antigenemia, and nucleic acid quantification can available for monitoring of HCMV infection in transplant recipients, even though all these methods have their own limitations $[79,82]$. Interestingly, one recent study assessed the in vivo pattern of blood HCMV miRNAs in SOT patients, and found that a number of HCMV-encoded miRNAs were existed in recipients with HCMV infection in variable relative abundance, and could be further evaluated as CMV infection biomarkers. Notably, statistical analysis revealed that the levels of miR-UL22a-5p could be used to independently predict the reactivation of HCMV upon discontinuation of antiviral treatment. More importantly, bioinformatics analyzes combined with further in vitro studies revealed that miR-UL22a-5p could interfere the translation of host genes that involved in antigen presentation and C-MYC [83]. Collectively, these results demonstrated the HCMV miRNAs may have complex effects on viral pathogenesis and have the potential as novel molecular markers for the prognosis of patients undergoing SOT. Like the pathological role of HCMV in SOT recipients, congenital cytomegalovirus infection is a main etiological reason of central nervous system and sensory disturbance that lead to neurodevelopmental disabilities [76]. Nevertheless, the clinical relevance between HCMV-encoded miRNAs and congenital HCMV infections has rarely been reported. Lately, Kawano et al. compared the plasma levels of miR-UL-112-3p, miR-US25-1-5p, and miR-US25-2-5p in the plasma of patients with congenital HCMV infection that with and without clinical symptom. Ultimately, two of the three examined HCMV miRNAs, including miR-US25-1-5p and miR-US25-2-5p, showed higher plasma levels in some patients with abnormal brain imaging as compared with patients with normal brain imaging [84]. This novel study of circulating HCMV miRNA expression profiles provides unique insight into the identification and evaluation the utility of extracellular HCMV miRNAs as non-invasive molecular marker of congenital HCMV infection. In addition, further mechanism studies indicated that these altered circulating viral miRNAs in congenital HCMV infection may also benefit for antiviral treatment. Increased levels of HCMV-encoded miRNAs in circulating were also found to be closely associated with cardiovascular disease, in particular, acute aortic dissection (AAD). AAD is one of the most catastrophic cardiovascular diseases with high mortality and misdiagnosis rate. However, none useful symptoms and techniques can be available to detect this disease immediate because of the insufficient sensitivity and specificity [85]. In a very recent study, the concentrations of miR-US33-5p were found to be markedly elevated in the plasma of AAD patients as compared with corresponding controls. ROC curve analyzes revealed that AUC of plasma miR-US33-5p for discriminating AAD from controls was 0.815 , with a relative high sensitivity and specificity [86]. These data indicate that detection of miR-US33-5p in circulation may helpful for diagnosing of AAD, moreover, altered miR-US33-5p may also suggest a potential pathological role of HCMV in the onset and progression of AAD.

To improve the understanding of both the diagnostic usefulness and pathological function of HCMV-encoded miRNAs within the context of HCMV infectious disease, our lab also explored the viral miRNA expression pattern in chronic hepatitis B virus (HBV) patients and oral lichen planus patients as well as their corresponding controls, respectively. Chronic hepatitis B is an infectious disease that with the highest prevalence in China. Currently, interferon $\alpha$ (IFN $\alpha)$ is a commonly used drug for acute or chronic HBV infection therapy in clinical. However, only a partial of CHB patients (about 25\% 40\%) can acquire a long-term sustained remission after IFN $\alpha$ treatment. Thus, novel and non-invasive biomarkers are urgently needed to evaluate the effectiveness of IFN $\alpha$ therapy would be benefited for patients' medication. To test the ability of serum HCMV-encoded miRNAs can be served as circulating marker to predict the effectiveness of IFN $\alpha$ therapy in patients with $\mathrm{CHB}$, the expression pattern of $13 \mathrm{HCMV}$-encoded miRNAs were firstly assessed in the serum of $\mathrm{CHB}$ patients who were responsive to IFN $\alpha$ treatment or not by qRT-PCR assays. Accordingly, two of the 13 examined HCMV miRNAs, including miR-US4-1 and 
miR-UL-148D, were identified to be markedly dysregulated in $\mathrm{CHB}$ patients who were responsive or not responsive to IFN $\alpha$ treatment [87]. Of note, subsequent perspective study revealed that detection of serum miR-US4-1 alone could accurately predict the effectiveness of IFN $\alpha$ therapy for $\mathrm{CHB}$ [87]. These results indicated that measurement of the levels of HCMV-encoded miRNA in serum of CHB patients may reflect the treatment effectiveness of IFNa. Furthermore, the above results may also indicate that HCMV and HCMV-encoded miRNAs participate in the pathological process of IFN $\alpha$ resistance in CHB patients. Abnormal pattern of HCMV-encoded miRNAs was also observed in the plasma of patients with oral lichen planus (OLP). OLP is a type of $\mathrm{T}$ cell-mediated autoimmune disease with the etiology remains unclarified currently. Moreover, objective criterions for diagnosing and subtype classification of OLP are still lacking. In one of our recent research, we investigated and compared the expression patterns of HCMV-encoded miRNA in plasma from patients with OLP and control subjects, respectively [35]. Consequently, a panel of five HCMV miRNAs were obviously upregulated in OLP patients as compared with controls. Of note, by comparing the plasma levels of the five miRNAs in the subtypes of OLP patients, including reticular OLP and erosive OLP, we found that the miR-UL112-3p and miR-UL36-5p were markedly increased in both reticular OLP and erosive OLP patients, while miR-UL22a-5p and miR-UL148D were statistically elevated in patients with erosive OLP. In addition, the plasma levels of miR-UL59 were merely increased in reticular OLP patients [35]. These data implied that the altered HCMV miRNAs in circulation may not only be used as potential biomarkers for OLP, but also have the ability to discriminate the distinct subtypes of OLP. Subsequently, to elucidate the possible pathophysiological role of the altered HCMV-encoded miRNAs in OLP, a combination of computational prediction, luciferase target gene reporter assay and western blot assay was applied to testify the potential targets of the five miRNAs. Among the targets, the cytomegalovirus UL16-binding protein 1 (ULBP1), was confirmed as a direct target gene of miR-UL59 [35]. Although the exact function of miR-UL59/ULBP1 regulation axis is currently unknown, the inhibition of ULBP1 protein which was implied in mediating the killing of virus infected cells by NK cells would result in decreased NK cell killing. Collectively, these preliminarily results may not only provide novel insight into the interaction between HCMV and OLP, but also warrants additional investigation in the mechanism underlying the process of OLP.

Currently, the aberrant expression patterns of HCMVencoded miRNAs in host circulation blood were still in their early stages. However, it is important in raising the possibility that the alterations of specific circulating HCMV-encoded miRNAs may provide diagnostic and prognostic information about HCMV infection and promise to be rich in biological information that will improve our understanding of the possible influence of virus miRNAs on HCMV-associated patient outcomes.

\section{Future perspectives and conclusions}

The discovery of viruses-encoded miRNAs leads to a surprising breakthrough in the realm of the interaction between viruses and their hosts, and also expand our knowledge about fundamental aspects of gene regulation through the action of exogenous miRNAs. Presently, even though much progress on understanding the biological functions of HCMV-encoded miRNAs has been made in the last two decades, the pathophysiological functions of HCMV in host diseases remains obscure, and the study area of HCMV and HCMV-encoded miRNAs still at a very preliminary stage. HCMV is widely distributed in almost all human populations and might reactivate periodically from latency, and the expression of HCMV-encoded miRNAs is observed in all phases of viral life cycle and can interfere both viral and host genes to facilitate viral immunoevasion, viral replication, viral latency, host cell survival and apoptosis, and innate and adaptive immunity. However, the functions of majority of the HCMV-encoded miRNAs have not been fully elucidated, and more target genes of HCMVencoded miRNAs are warranted to be investigated to comprehensively clarify the complex interaction between HCMV and cell host. In particular, in-depth exploration of HCMV miRNAs will help to delve into the potential mechanisms that related with HCMV infection and pathogenesis. Indeed, existing reports on the HCMV miRNAs expression pattern were characterized only in a few types of virus susceptible cells such as fibroblasts and THP-1 monocyte, and the functional investigations of the aberrant miRNAs were mainly focused on few specific miRNAs, such as miR-UL112-3p and miR-UL148D. Thus, future studies by using different cell models and technologies are urgently needed towards the clear definition of other HCMV miRNAs targetome and their functional relevance in viral infection, latency and pathogenesis, and the results will help us to truly understand their functional significance.

Existing evidence have uncovered the altered HCMV miRNAs expression pattern between patients with glioblastoma, oral disease and healthy controls. Theoretically, understanding how HCMV miRNA influence disease progression will provide novel insights into implications for the development of novel therapeutic interventions and improve outcomes for HCMV infectious disease. Unfortunately, the HCMV-encoded miRNAs are currently restricted to just HCMV-associated glioblastoma and oral disease. Given the fact that HCMV has a prevalence of $90 \sim 100 \%$ within the human population, it is reasonable to speculate that HCMV 
miRNAs also play pivot pathophysiological roles in other diseases development. Therefore, deep investigations into the relationship between the altered HCMV miRNAs and other disease as well as the potential mechanism underlying with disease progression mediated by HCMV miRNAs would benefit their clinical translational in $\mathrm{HCMV}$-associated diseases diagnosing and therapizing.

The presence of altered HCMV-encoded miRNAs in the blood circulation of different types of HCMV-associated diseases raises a novel possible of circulating HCMV miRNAs to become a promise minimal-invasive diagnostic and prognostic tool in coming future. In general, a working model for screening of circulating HCMV-encoded miRNA-based biomarkers for diseases can be separated into two phases: (i) initial screening of dysregulated HCMV-encoded miRNAs in individual or pooled samples using next-generation sequencing technology, commercial microarray or qRT-PCR; and (ii) qRT-PCR validation in a large number of individual samples arranged in multiple training and verification sets. By applying this working model, to date, the serum or plasma signatures of HCMV-encoded miRNAs have been reported in patients with essential hypertension, type 2 diabetes, glioblastoma, solid organ transplantation, congenital HCMV infection, acute aortic dissection, chronic hepatitis $\mathrm{B}$ and oral lichen planus, while a number of miRNAs such as miR-UL112-3p, miR-US33-5p, miR-US4-1, miR-UL22a-5p, miR-UL148D and miR-UL59 were confirmed to be markedly dysregulated between those patients and their corresponding controls. Notably, of these dysregulated circulating HCMV miRNAs, miR-UL112-3p, miR-UL22a-5p and miR-UL148D shared similarly alteration in two or three different types of disease, and which will attenuate their diagnostic specificity as candidate markers for HCMV-associated disease. Thus, large-scale efforts to identify disease specific altered miRNAs and/or miRNA-panel should be conducted and emphases in future researches. On the other hand, all of the above-mentioned studies enrolled a relatively small sample size of cases and controls that from only one center, therefore, future systematical analyzes of HCMV-encoded miRNA expression pattern from multiethnic and multicentric with large number cases and controls are urgently required.

The discovery of HCMV-encoded miRNAs in the blood circulation of patients with HCMV infection also open up new insight into the investigation on HCMV-host interactions and provide potential therapeutic interventions for the treatment of HCMV infectious disease. In the past decade, accumulating evidence has uncovered the presence of cell-free miRNAs in extracellular exosomes in human circulation and other body fluids [88]. More interestingly, circulating miRNAs in blood that secreted actively through exosomes may function as an intercellular and interorgan communication system in the body
$[33,89,90]$. Indeed, several recent reports from our labs and others have demonstrated that circulating miRNAs were transferred through exosomes may participate in cell-cell communication by targeting downstream mRNA genes in surrounding or distant recipient cells both in vitro and in vivo [91-94]. This is of especially meaningful as virus-encoded miRNAs can also be encapsulated into exosomes and transferred into different host cells revealing a paracrine nature of virus-encoded miRNAs. For instance, EBV-positive B-lymphoblastoid cells can secrete exosomes that containing EBV-encoded miRNAs, moreover, secreted EBV miRNAs can be transferred to EBV-negative host cells through exosome and leads a downregulation of the chemokine CXC ligand 11 [95]. One subsequent study also found that the EBV-encoded miRNAs can be secreted by EBV-infected NPC cells through exosomes, and the exosome-encapsulated EBV miRNAs may involve in the crosstalk between tumor cells and nonmalignant cells [96]. Interestingly, another contemporaneous study confirmed that exosomes released from NPC cells can enter into many types of host cells including epithelial, endothelial, and fibroblast cells, and modulating the tumor microenvironment to influence the cells growth by activating the ERK and AKT signaling pathways [97]. Besides EBV miRNAs, other herpesvirus miRNAs such as KSHV-encoded miRNAs were also observed in exosomes which were derived from KSHV positive cell lines and malignancies [19, 98, 99]. Moreover, following mechanism study revealed that KSHV-positive cells specifically deliver the KSHV-encoded miRNAs to neighboring cells through exosomes. Importantly, the virus miRNAs carried by exosome can regulate the aerobic glycolysis metabolic reprogramming of non-infected neighboring cells as well as metabolic niche and contribute to viral fitness [100]. Collectively, the above findings provide important insight into the function of exosome-based virus miRNAs in manipulating host cellular functions, subverting immune responses and their possible roles in the viral pathology.

Till date, little is known about the detailed distribution and the role of circulating HCMV miRNAs. Nevertheless, our recent preliminary study revealed that the majority of plasma homv-miR-UL59 was encapsulated in the exosome vesicles during HCMV latency, which provide a novel understanding that altered HCMV-encoded miRNAs in the blood circulation might be actively secreted from HCMV infected or inflammatory cells in which the virus resides via exosomes, and utilize as informatic vehicles facilitating virus-cell communication [35]. Additionally, given the ability of exosomes can transfer small molecular from a host cell to recipient cell, our study also highlights an exciting potential of sequence specific RNA molecules in the treatment of viral infections. Indeed, exosome-based miRNAs communication provides viruses with a novel mechanism and elegant tool for non-infectious transfer of genetic material 
without production of new viral particles, due to their nonimmunogenic nature to evade the host immune system. Therefore, future studies focus on studying the function of these exosomes-containing HCMV miRNAs in the circulation would undoubtedly boost the research and development of a more useful therapeutic strategy to combat HCMV-associated disease and to better predict the prognosis. In addition, other studies on developing therapeutic interventions by manipulation the expression of viral miRNAs through exosomes that derived from genetically engineered cells delivery of small RNAs are also warranted in the future.

\begin{abstract}
Abbreviations
AAD: acute aortic dissection; ANT3: adenine nucleotide translocator 3; AUC: the area under the ROC curve; BKV: BK virus; BL: Burkitt's lymphoma; CDC25B: cell division cycle 25B; CHB: chronic hepatitis B; EBV: Epstein-Barr virus; elF4A1: eukaryotic translation initiation factor 4A1; EVD: Ebola virus disease; GBM: glioblastoma; GC: gastric carcinoma; HCMV: human cytomegalovirus; HELs: human embryo lung fibroblasts; HFF: human foreskin fibroblasts; HL: Hodgkin's lymphoma; IE: immediate early; IER5: immediate early response gene 5; IEX-1: immediate early gene X-1; IFNa: interferon a; IL32: Interleukin-32; JCPyV: Polyomavirus JC; KSHV: Kaposi sarcoma-associated herpesvirus; MICB: major histocompatibility complex class I-related chain B; miRNA: microRNA; MV: microvesicles; NNKTL: Nasal natural killer/T-cell lymphoma; NPC: nasopharyngeal carcinoma; OLP: Oral lichen planus; PBLs: peripheral blood leukocytes; PVs: Papillomaviruses; QARS: GlutaminyltRNA Synthetase; qRT-PCR: quantitative reverse transcription polymerase chain reaction; ROC: receiver operating characteristic curve; TLR: Toll-Like Receptor; ULBP1: UL16-binding protein 1
\end{abstract}

\section{Acknowledgements}

Not applicable.

\section{Funding}

This work was supported by grants from National Natural Science Foundation of China (no. 81772282 and 81672102), Foundation of Jiangsu Provincial Medical Youth Talent (QNRC2016893), Fund of State Key Laboratory of Analytical Chemistry for Life Science (no. 5431ZZXM1601), and National Basic Research Program of China (no. 2014CB542300).

\section{Availability of data and materials}

Not applicable.

\section{Authors' contributions}

C.W. wrote the manuscript. C.Z. and J.J.W. revised the manuscript. All authors read and approved the final manuscript.

\section{Ethics approval and consent to participate}

Not applicable.

\section{Consent for publication}

All the authors agree to the publication.

\section{Competing interests}

The authors declare no competing interests.

\section{Publisher's Note}

Springer Nature remains neutral with regard to jurisdictional claims in published maps and institutional affiliations.

Received: 27 September 2018 Accepted: 15 March 2019 Published online: 02 October 2019

\section{References}

1. Kozomara A. Griffiths-Jones S: miRBase: annotating high confidence microRNAs using deep sequencing data. Nucleic Acids Res. 2014;42:D68-73.
2. Friedman RC, Farh KK, Burge CB, Bartel DP. Most mammalian mRNAs are conserved targets of microRNAs. Genome Res. 2009;19:92-105.

3. Bartel DP. MicroRNAs: genomics, biogenesis, mechanism, and function. Cell. 2004;116:281-97.

4. Sullivan CS, Ganem D. MicroRNAs and viral infection. Mol Cell. 2005;20:3-7.

5. Powdrill MH, Desrochers GF, Singaravelu R, Pezacki JP. The role of microRNAs in metabolic interactions between viruses and their hosts. Curr Opin Virol. 2016;19:71-6.

6. Tudor S, Giza DE, Lin HY, Fabris L, Yoshiaki K, D'Abundo L, Toale KM, Shimizu M, Ferracin M, Challagundla KB, et al. Cellular and Kaposi's sarcomaassociated herpes virus microRNAs in sepsis and surgical trauma. Cell Death Dis. 2014;5:e1559.

7. Chen X, Ba Y, Ma L, Cai X, Yin Y, Wang K, Guo J, Zhang Y, Chen J, Guo X, et al. Characterization of microRNAs in serum: a novel class of biomarkers for diagnosis of cancer and other diseases. Cell Res. 2008;18:997-1006.

8. Komabayashi Y, Kishibe K, Nagato T, Ueda S, Takahara M, Harabuchi Y. Circulating Epstein-Barr virus-encoded micro-RNAs as potential biomarkers for nasal natural killer/T-cell lymphoma. Hematol Oncol. 2017;35:655-63.

9. Kawano Y, Iwata S, Kawada J, Gotoh K, Suzuki M, Torii Y, Kojima S, Kimura H, Ito Y. Plasma viral microRNA profiles reveal potential biomarkers for chronic active Epstein-Barr virus infection. J Infect Dis. 2013;208:771-9.

10. Li JY, McNicholas K, Yong TY, Rao N, Coates PT, Higgins GD, Carroll RP, Woodman RJ, Michael MZ, Gleadle JM. BK virus encoded microRNAs are present in blood of renal transplant recipients with BK viral nephropathy. Am J Transplant. 2014;14:1183-90.

11. Hirai N, Wakisaka N, Kondo S, Aga M, Moriyama-Kita M, Ueno T, Nakanishi Y, Endo K, Sugimoto $\mathrm{H}$, Murono $\mathrm{S}$, et al. Potential interest in circulating miR-BART17-5p as a post-treatment biomarker for prediction of recurrence in Epstein-Barr virus-related nasopharyngeal carcinoma. PLoS One. 2016;11:e0163609.

12. Li H, Liu S, Hu J, Luo X, Li N. A MB, Cao Y: Epstein-Barr virus lytic reactivation regulation and its pathogenic role in carcinogenesis. Int J Biol Sci. 2016;12: 1309-18.

13. Jha HC, Pei Y, Robertson ES. Epstein-Barr virus: diseases linked to infection and transformation. Front Microbiol. 2016;7:1602.

14. Cai X, Schafer A, Lu S, Bilello JP, Desrosiers RC, Edwards R, Raab-Traub N, Cullen BR. Epstein-Barr virus microRNAs are evolutionarily conserved and differentially expressed. PLoS Pathog. 2006;2:e23.

15. Ferrajoli A, Ivan C, Ciccone M, Shimizu M, Kita Y, Ohtsuka M, D'Abundo L, Qiang J, Lerner S, Nouraee N, et al. Epstein-Barr virus MicroRNAs are expressed in patients with chronic lymphocytic leukemia and correlate with overall survival. EBioMedicine. 2015;2:572-82.

16. Jiang $C$, Chen J, Xie S, Zhang L, Xiang Y, Lung M, Kam NW, Kwong DL, Cao S, Guan XY. Evaluation of circulating EBV microRNA BART2-5p in facilitating early detection and screening of nasopharyngeal carcinoma. Int J Cancer. 2018.

17. Zhang G, Zong J, Lin S, Verhoeven RJ, Tong S, Chen Y, Ji M, Cheng W, Tsao SW, Lung $M$, et al. Circulating Epstein-Barr virus microRNAs miR-BART7 and miRBART13 as biomarkers for nasopharyngeal carcinoma diagnosis and treatment. Int J Cancer. 2015:136:E301-12

18. Treece AL, Duncan DL, Tang W, Elmore S, Morgan DR, Dominguez RL, Speck O, Meyers MO, Gulley ML. Gastric adenocarcinoma microRNA profiles in fixed tissue and in plasma reveal cancer-associated and Epstein-Barr virus-related expression patterns. Lab Investig. 2016:96:661-71.

19. Hoshina S, Sekizuka T, Kataoka M, Hasegawa H, Hamada H, Kuroda M, Katano $\mathrm{H}$. Profile of Exosomal and intracellular microRNA in gammaherpesvirus-infected lymphoma cell lines. PLoS One. 2016;11:e0162574.

20. Fuentes-Mattei E, Giza DE, Shimizu M, Ivan C, Manning JT, Tudor S, Ciccone $M$, Kargin OA, Zhang $X$, Mur $P$, et al. Plasma viral miRNAs indicate a high prevalence of occult viral infections. EBioMedicine. 2017;20:182-92.

21. Giovannelli I, Martelli F, Repice A, Massacesi L, Azzi A, Giannecchini S. Detection of JCPyV microRNA in blood and urine samples of multiple sclerosis patients under natalizumab therapy. J Neuro-Oncol. 2015;21:666-70.

22. Chirayil R, Kincaid RP, Dahlke C, Kuny CV, Dalken N, Spohn M, Lawson B, Grundhoff A, Sullivan CS. Identification of virus-encoded microRNAs in divergent papillomaviruses. PLoS Pathog. 2018;14:e1007156.

23. Chen Z, Liang $\mathrm{H}$, Chen $\mathrm{X}$, Ke $Y$, Zhou Z, Yang M, Zen K, Yang R, Liu C, Zhang CY. An Ebola virus-encoded microRNA-like fragment serves as a biomarker for early diagnosis of Ebola virus disease. Cell Res. 2016;26:380-3.

24. Grey F, Meyers H, White EA, Spector DH, Nelson J. A human cytomegalovirus-encoded microRNA regulates expression of multiple viral genes involved in replication. PLoS Pathog. 2007;3:e163. 
25. Stark TJ, Arnold JD, Spector DH, Yeo GW. High-resolution profiling and analysis of viral and host small RNAs during human cytomegalovirus infection. J Virol. 2012;86:226-35.

26. Grey F, Tirabassi R, Meyers H, Wu G, McWeeney S, Hook L, Nelson JA. A viral microRNA down-regulates multiple cell cycle genes through mRNA 5'UTRs. PLoS Pathog. 2010;6:e1000967.

27. Nachmani D, Lankry D, Wolf DG, Mandelboim O. The human cytomegalovirus microRNA miR-UL112 acts synergistically with a cellular microRNA to escape immune elimination. Nat Immunol. 2010;11:806-13.

28. Dhuruvasan K, Sivasubramanian G, Pellett PE. Roles of host and viral microRNAs in human cytomegalovirus biology. Virus Res. 2011;157:180-92.

29. Kim S, Lee S, Shin J, Kim Y, Evnouchidou I, Kim D, Kim YK, Kim YE, Ahn JH, Riddell SR, et al. Human cytomegalovirus microRNA miR-US4-1 inhibits CD8(+) T cell responses by targeting the aminopeptidase ERAP1. Nat Immunol. 2011:12:984-91.

30. Hook LM, Grey F, Grabski R, Tirabassi R, Doyle T, Hancock M, Landais I, Jeng S, McWeeney S, Britt W, Nelson JA. Cytomegalovirus miRNAs target secretory pathway genes to facilitate formation of the virion assembly compartment and reduce cytokine secretion. Cell Host Microbe. 2014;15: 363-73

31. Shen $K$, Xu L, Chen D, Tang W, Huang Y. Human cytomegalovirus-encoded miR-UL112 contributes to HCMV-mediated vascular diseases by inducing vascular endothelial cell dysfunction. Virus Genes. 2018;54:172-81.

32. Li S, Zhu J, Zhang W, Chen Y, Zhang K, Popescu LM, Ma X, Lau WB, Rong R, $Y u X$, et al. Signature microRNA expression profile of essential hypertension and its novel link to human cytomegalovirus infection. Circulation. 2011;124: 175-84.

33. Chen $X$, Liang $H$, Zhang J, Zen $K$, Zhang CY. Secreted microRNAs: a new form of intercellular communication. Trends Cell Biol. 2012;22:125-32.

34. Lagana A, Russo F, Veneziano D, Bella SD, Giugno R, Pulvirenti A, Croce CM, Ferro A. Extracellular circulating viral microRNAs: current knowledge and perspectives. Front Genet. 2013:4:120.

35. Ding $M$, Wang $X$, Wang C, Liu X, Zen K, Wang W, Zhang CY, Zhang C. Distinct expression profile of HCMV encoded miRNAs in plasma from oral lichen planus patients. J Transl Med. 2017;15:133.

36. Fannin Rider PJ, Dunn W, Yang E, Liu F. Human cytomegalovirus microRNAs. Curr Top Microbiol Immunol. 2008;325:21-39.

37. Kincaid RP, Sullivan CS. Virus-encoded microRNAs: an overview and a look to the future. PLoS Pathog. 2012:8:e1003018.

38. Grey F, Nelson J. Identification and function of human cytomegalovirus microRNAs. J Clin Virol. 2008;41:186-91.

39. Pfeffer S, Sewer A, Lagos-Quintana M, Sheridan R, Sander C, Grasser FA, van Dyk LF, Ho CK, Shuman S, Chien M, et al. Identification of microRNAs of the herpesvirus family. Nat Methods. 2005;2:269-76.

40. Grey F, Antoniewicz A, Allen E, Saugstad J, McShea A, Carrington JC, Nelson J. Identification and characterization of human cytomegalovirus-encoded microRNAs. J Virol. 2005;79:12095-9.

41. Dunn W, Trang P, Zhong Q, Yang E, van Belle C, Liu F. Human cytomegalovirus expresses novel microRNAs during productive viral infection. Cell Microbiol. 2005;7:1684-95.

42. Fu M, Gao Y, Zhou Q, Zhang Q, Peng Y, Tian K, Wang J, Zheng X. Human cytomegalovirus latent infection alters the expression of cellular and viral microRNA. Gene. 2014;536:272-8.

43. Shen ZZ, Pan X, Miao LF, Ye HQ, Chavanas S, Davrinche C, McVoy M, Luo $\mathrm{MH}$. Comprehensive analysis of human cytomegalovirus microRNA expression during lytic and quiescent infection. PLoS One. 2014;9:e88531.

44. Ulasov IV, Kaverina NV, Ghosh D, Baryshnikova MA, Kadagidze ZG, Karseladze Al, Baryshnikov AY, Cobbs CS. CMV70-3P miRNA contributes to the CMV mediated glioma stemness and represents a target for glioma experimental therapy. Oncotarget. 2017:8:25989-99.

45. Liang Q, Wang K, Wang B, Cai Q. HCMV-encoded miR-UL112-3p promotes glioblastoma progression via tumour suppressor candidate 3. Sci Rep. 2017; 7:44705.

46. Deshpande RP, Panigrahi M, Y BVKC, Babu PP: Profiling of microRNAs modulating cytomegalovirus infection in astrocytoma patients. Neurol Sci 2018.

47. Zhong S, Naqvi A, Bair E, Nares S, Khan AA. Viral MicroRNAs identified in human dental pulp. J Endod. 2017:43:84-9.

48. Naqvi AR, Seal A, Shango J, Brambila MF, Martinez G, Chapa G, Hasan S, Yadavalli T, Jaishankar D, Shukla D, Nares S. Herpesvirus-encoded microRNAs detected in human gingiva alter host cell transcriptome and regulate viral infection. Biochim Biophys Acta. 1861;2018:497-508.
49. Stern-Ginossar N, Elefant N, Zimmermann A, Wolf DG, Saleh N, Biton M, Horwitz E, Prokocimer Z, Prichard M, Hahn G, et al. Host immune system gene targeting by a viral miRNA. Science. 2007;317:376-81.

50. Huang Y, Qi Y, Ma Y, He R, Ji Y, Sun Z, Ruan Q. The expression of interleukin-32 is activated by human cytomegalovirus infection and down regulated by hcmv-miR-UL112-1. Virol J. 2013;10:51.

51. Romania P, Cifaldi L, Pignoloni B, Starc N, D'Alicandro V, Melaiu O, Li Pira G, Giorda E, Carrozzo R, Bergvall M, et al. Identification of a genetic variation in ERAP1 aminopeptidase that prevents human cytomegalovirus miR-UL1125p-mediated Immunoevasion. Cell Rep. 2017;20:846-53.

52. Stern-Ginossar N, Saleh N, Goldberg MD, Prichard M, Wolf DG, Mandelboim O. Analysis of human cytomegalovirus-encoded microRNA activity during infection. J Virol. 2009:83:10684-93.

53. Qi M, Qi Y, Ma Y, He R, Ji Y, Sun Z, Ruan Q. Over-expression of human cytomegalovirus miR-US25-2-3p downregulates elF4A1 and inhibits HCMV replication. FEBS Lett. 2013;587:2266-71.

54. Pavelin J, Reynolds N, Chiweshe S, Wu G, Tiribassi R, Grey F. Systematic microRNA analysis identifies ATP6V0C as an essential host factor for human cytomegalovirus replication. PLoS Pathog. 2013;9:e1003820.

55. Jiang S, Qi Y, He R, Huang Y, Liu Z, Ma Y, Guo X, Shao Y, Sun Z, Ruan Q. Human cytomegalovirus microRNA miR-US25-1-5p inhibits viral replication by targeting multiple cellular genes during infection. Gene. 2015;570:108-14.

56. Guo X, Qi Y, Huang Y, Liu Z, Ma Y, Shao Y, Jiang S, Sun Z, Ruan Q. Human cytomegalovirus miR-US33-5p inhibits viral DNA synthesis and viral replication by down-regulating expression of the host Syntaxin3. FEBS Lett. 2015;589:440-6.

57. Jiang S, Huang Y, Qi Y, He R, Liu Z, Ma Y, Guo X, Shao Y, Sun Z, Ruan Q. Human cytomegalovirus miR-US5-1 inhibits viral replication by targeting geminin mRNA. Virol Sin. 2017;32:431-9.

58. Stinski MF, Meier JL: Immediate-early viral gene regulation and function. In Human Herpesviruses: Biology, Therapy, and Immunoprophylaxis. Edited by Arvin A, Campadelli-Fiume G, Mocarski E, Moore PS, Roizman B, Whitley R, Yamanishi K. Cambridge; 2007.

59. Murphy E, Vanicek J, Robins H, Shenk T, Levine AJ. Suppression of immediate-early viral gene expression by herpesvirus-coded microRNAs: implications for latency. Proc Natl Acad Sci U S A. 2008;105:5453-8.

60. Lau B, Poole E, Van Damme E, Bunkens L, Sowash M, King H, Murphy E, Wills M, Van Loock M, Sinclair J. Human cytomegalovirus miR-UL112-1 promotes the down-regulation of viral immediate early-gene expression during latency to prevent T-cell recognition of latently infected cells. J Gen Virol. 2016;97:2387-98.

61. Pan C, Zhu D, Wang Y, Li L, Li D, Liu F, Zhang CY, Zen K. Human cytomegalovirus miR-UL148D facilitates latent viral infection by targeting host cell immediate early response gene 5. PLoS Pathog. 2016;12:e1006007.

62. Huang Y, Chen D, He J, Cai J, Shen K, Liu X, Yang X, Xu L. Hcmv-miR-UL112 attenuates NK cell activity by inhibition type I interferon secretion. Immunol Lett. 2015;163:151-6.

63. Landais I, Pelton C, Streblow D, DeFilippis V, McWeeney S, Nelson JA. Human cytomegalovirus miR-UL112-3p targets TLR2 and modulates the TLR2/IRAK1/NFkappaB signaling pathway. PLoS Pathog. 2015;11:e1004881.

64. Billstrom Schroeder M, Worthen GS. Viral regulation of RANTES expression during human cytomegalovirus infection of endothelial cells. J Virol. 2001; 75:3383-90

65. Kim Y, Lee S, Kim S, Kim D, Ahn JH, Ahn K. Human cytomegalovirus clinical strain-specific microRNA miR-UL 148D targets the human chemokine RANTES during infection. PLoS Pathog. 2012;8:e1002577.

66. Lau B, Poole E, Krishna B, Sellart I, Wills MR, Murphy E, Sinclair J. The expression of human cytomegalovirus MicroRNA MiR-UL148D during latent infection in primary myeloid cells inhibits Activin A-triggered secretion of IL6. Sci Rep. 2016;6:31205

67. Chen J, Xia S, Yang X, Chen H, Li F, Liu F, Chen Z. Human cytomegalovirus encoded miR-US25-1-5p attenuates CD147/EMMPRIN-mediated early antiviral response. Viruses. 2017:9.

68. Arlt A, Kruse ML, Breitenbroich M, Gehrz A, Koc B, Minkenberg J, Folsch UR, Schafer $\mathrm{H}$. The early response gene IEX-1 attenuates NF-kappaB activation in 293 cells, a possible counter-regulatory process leading to enhanced cell death. Oncogene. 2003;22:3343-51.

69. Wang YP, Qi Y, Huang YJ, Qi ML, Ma YP, He R, Ji YH, Sun ZR, Ruan Q. Identification of immediate early gene $\mathrm{X}-1$ as a cellular target gene of hcmv-mir-UL148D. Int J Mol Med. 2013;31:959-66. 
70. Babu SG, Pandeya A, Verma N, Shukla N, Kumar RV, Saxena S. Role of HCMV miR-UL70-3p and miR-UL148D in overcoming the cellular apoptosis. Mol Cell Biochem. 2014;393:89-98.

71. Jang JY, Choi Y, Jeon YK, Aung KC, Kim CW. Over-expression of adenine nucleotide translocase 1 (ANT1) induces apoptosis and tumor regression in vivo. BMC Cancer. 2008:8:160.

72. Yang Z, Cheng W, Hong L, Chen W, Wang Y, Lin S, Han J, Zhou H, Gu J. Adenine nucleotide (ADP/ATP) translocase 3 participates in the tumor necrosis factor induced apoptosis of MCF-7 cells. Mol Biol Cell. 2007;18: 4681-9.

73. Guo X, Huang Y, Qi Y, Liu Z, Ma Y, Shao Y, Jiang S, Sun Z, Ruan Q. Human cytomegalovirus miR-UL36-5p inhibits apoptosis via downregulation of adenine nucleotide translocator 3 in cultured cells. Arch Virol. 2015;160:2483-90.

74. Ko YG, Kim EY, Kim T, Park H, Park HS, Choi EJ, Kim S. Glutamine-dependent antiapoptotic interaction of human glutaminyl-tRNA synthetase with apoptosis signal-regulating kinase 1. J Biol Chem. 2001;276:6030-6.

75. Fan J, Zhang W, Liu Q. Human cytomegalovirus-encoded miR-US25-1 aggravates the oxidised low density lipoprotein-induced apoptosis of endothelial cells. Biomed Res Int. 2014;2014:531979.

76. Pass RF, Arav-Boger R: Maternal and fetal cytomegalovirus infection: diagnosis, management, and prevention. F1000Res 2018, 7:255.

77. Griffiths PD. Burden of disease associated with human cytomegalovirus and prospects for elimination by universal immunisation. Lancet Infect Dis. 2012; 12:790-8.

78. Drew WL. Laboratory diagnosis of cytomegalovirus infection and disease in immunocompromised patients. Curr Opin Infect Dis. 2007;20:408-11.

79. Dioverti MV, Razonable RR. Cytomegalovirus. Microbiol Spectr. 2016;4

80. Spector SA, Merrill R, Wolf D, Dankner WM. Detection of human cytomegalovirus in plasma of AIDS patients during acute visceral disease by DNA amplification. J Clin Microbiol. 1992;30:2359-65.

81. Mohammad AA, Rahbar A, Lui WO, Davoudi B, Catrina A, Stragliotto G, Mellbin L, Hamsten A, Ryden L, Yaiw KC, Soderberg-Naucler C. Detection of circulating homv-miR-UL112-3p in patients with glioblastoma, rheumatoid arthritis, diabetes mellitus and healthy controls. PLoS One. 2014:9:e113740.

82. Dioverti MV, Razonable RR. Clinical utility of cytomegalovirus viral load in solid organ transplant recipients. Curr Opin Infect Dis. 2015;28:317-22.

83. Lisboa LF, Egli A, O'Shea D, Asberg A, Hartmann A, Rollag H, Pang XL, Tyrrell DL, Kumar D, Humar A. Hcmv-miR-UL22A-5p: a biomarker in transplantation with broad impact on host gene expression and potential immunological implications. Am J Transplant. 2015;15:1893-902.

84. Kawano Y, Kawada J, Kamiya Y, Suzuki M, Torii Y, Kimura H, Ito Y. Analysis of circulating human and viral microRNAs in patients with congenital cytomegalovirus infection. J Perinatol. 2016;36:1101-5.

85. Hiraya D, Sato A, Aonuma K. Circulating microRNAs as an emerging biomarker for acute aortic dissection diagnosis-comparing with prior biomarkers. J Thorac Dis. 2018;10:1186-9.

86. Dong J, Bao J, Feng R, Zhao Z, Lu Q, Wang G, Li H, Su D, Zhou J, Jing Q, Jing Z. Circulating microRNAs: a novel potential biomarker for diagnosing acute aortic dissection. Sci Rep. 2017;7:12784.

87. Pan Y, Wang N, Zhou Z, Liang H, Pan C, Zhu D, Liu F, Zhang CY, Zhang Y, Zen K. Circulating human cytomegalovirus-encoded HCMV-miR-US4-1 as an indicator for predicting the efficacy of IFNalpha treatment in chronic hepatitis B patients. Sci Rep. 2016;6:23007.

88. Liang H, Gong F, Zhang S, Zhang CY, Zen K, Chen X. The origin, function, and diagnostic potential of extracellular microRNAs in human body fluids. Wiley Interdiscip Rev RNA. 2014;5:285-300.

89. Chen $\mathrm{X}$, Liang $\mathrm{H}$, Zhang J, Zen $\mathrm{K}$, Zhang $\mathrm{CY}$. Horizontal transfer of microRNAs: molecular mechanisms and clinical applications. Protein Cell. 2012;3:28-37.

90. Huang-Doran I, Zhang CY, Vidal-Puig A. Extracellular vesicles: novel mediators of cell communication in metabolic disease. Trends Endocrinol Metab. 2017;28:3-18.

91. Skog J, Wurdinger T, van Rijn S, Meijer DH, Gainche L, Sena-Esteves M, Curry WT Jr, Carter BS, Krichevsky AM, Breakefield XO. Glioblastoma microvesicles transport RNA and proteins that promote tumour growth and provide diagnostic biomarkers. Nat Cell Biol. 2008;10:1470-6.

92. Zhang Y, Liu D, Chen X, Li J, Li L, Bian Z, Sun F, Lu J, Yin Y, Cai X, et al. Secreted monocytic miR-150 enhances targeted endothelial cell migration. Mol Cell. 2010;39:133-44

93. Li J, Zhang Y, Liu Y, Dai X, Li W, Cai X, Yin Y, Wang Q, Xue Y, Wang C, et al. Microvesicle-mediated transfer of microRNA-150 from monocytes to endothelial cells promotes angiogenesis. J Biol Chem. 2013;288:23586-96.
94. Pan Y, Liang H, Liu H, Li D, Chen X, Li L, Zhang CY, Zen K. Platelet-secreted microRNA-223 promotes endothelial cell apoptosis induced by advanced glycation end products via targeting the insulin-like growth factor 1 receptor. J Immunol. 2014;192:437-46.

95. Pegtel DM, Cosmopoulos K, Thorley-Lawson DA, van Eijndhoven MA, Hopmans ES, Lindenberg JL, de Gruijl TD, Wurdinger T, Middeldorp JM. Functional delivery of viral miRNAs via exosomes. Proc Natl Acad Sci U S A. 2010;107:6328-33.

96. Gourzones C, Gelin A, Bombik I, Klibi J, Verillaud B, Guigay J, Lang P, Temam $S$, Schneider $V$, Amiel $C$, et al. Extra-cellular release and blood diffusion of BART viral micro-RNAs produced by EBV-infected nasopharyngeal carcinoma cells. Virol J. 2010;7:271.

97. Meckes DG Jr, Shair KH, Marquitz AR, Kung CP, Edwards RH, Raab-Traub N. Human tumor virus utilizes exosomes for intercellular communication. Proc Natl Acad Sci U S A. 2010;107:20370-5.

98. Chugh PE, Sin SH, Ozgur S, Henry DH, Menezes P, Griffith J, Eron JJ, Damania B, Dittmer DP. Systemically circulating viral and tumor-derived microRNAs in KSHV-associated malignancies. PLoS Pathog. 2013;9:e1003484.

99. Naqvi AR, Shango J, Seal A, Shukla D, Nares S. Viral miRNAs Alter host cell miRNA profiles and modulate innate immune responses. Front Immunol. 2018;9:433.

100. Yogev O, Henderson S, Hayes MJ, Marelli SS, Ofir-Birin Y, Regev-Rudzki N, Herrero J, Enver T. Herpesviruses shape tumour microenvironment through exosomal transfer of viral microRNAs. PLoS Pathog. 2017;13:e1006524.
Ready to submit your research? Choose BMC and benefit from:

- fast, convenient online submission

- thorough peer review by experienced researchers in your field

- rapid publication on acceptance

- support for research data, including large and complex data types

- gold Open Access which fosters wider collaboration and increased citations

- maximum visibility for your research: over 100M website views per year

At BMC, research is always in progress.

Learn more biomedcentral.com/submissions 